\title{
Technology, Accessibility and Creativity in Popular Music Education
}

Ben Challis

There has been steady growth in the affordable music-technology market now for somewhere in the order of thirty years. During this period there has been a noticeable shift in emphasis from complex and often expensive resources to more accessible and affordable counterparts. It is now entirely feasible for the aspiring artist, composer or producer to draw upon any number of technological tools that have been designed to make all manner of processes achievable that would normally have called upon the expertise and skill of 'the professional'. Individuals who would not ordinarily have been in a position to gain the appropriate skills to perform many, if any, of all manner of complex musical and/or production tasks can often now achieve these same tasks through assisted means. For example, there is no longer an absolute prerequisite of being a highly trained or skilled musician to employ the realistic sounds of any number of common and exotic instruments. This is not only true within a context of composition, arranging and performance but also within many aspects of the production process. Sixty four-track studios with numerous off-board effects and sound processors are no longer the exclusive domain of successful commercial bands; the 'virtual-studio' has opened up this potential to anyone with enough space to house a relatively inexpensive home-computer.

However, it is an unfortunate truth that much of the impetus behind the design and manufacture of any new technology is driven by market forces. The knock-on effect is that most of the constraints that are imposed within the design process are derived from the perceptual and physical limits of the ordinary-user. In stark contrast to the notion of enabling the many, these same technologies often provide additional barriers to those who have extra-ordinary needs. As technology becomes smaller, so do the controls with which we interact with the system, sometimes to such a degree that smooth or successful interaction for those with less than average dexterity is an unlikely outcome. In spite of the accessibility options of some current software programmes, where 'real' becomes 'virtual' there is an expectation that the user is both fully-sighted and also in a position to freely manipulate complex on-screen graphics.

There is a simple yet important observation to make in that the majority of music-technology is quite simply not designed with disabled users in mind. A more concerning observation is that much of the equipment that is perhaps considered as 'industry standard', is also in common usage within schools, colleges and universities across the UK. Indeed, there is evidence to suggest that, within this, the variety of music-based software being used, for example, in secondary schools is actually very limited with certain packages being clearly favoured (noticeably Cubase and Sibelius) (Pitts and Kwami 2002). Many of these tools and technologies may well simply exaggerate the challenges already faced by music-learners and performers with disabilities. This does not mean that there cannot be any exciting developments that are specifically aimed at musicians and music-learners with disabilities; there are, and this article will describe and discuss the various merits of some of these novel technologies. In essence, this article is an attempt to provide a 'snapshot' of the current state of accessibility in music composition, performance and production. To 
place this in context though, it is first worth considering where the diverse array of music-making technology available to us today has evolved from.

\section{A Brief History}

A complete overview of the evolution of electronic performance and production technology is beyond the scope and purpose of this article. However, there have been key developments and aspects within this steady progression that are worthy of discussion when considering accessibility in this area. Perhaps most noticeable has been the general transition from analogue electronics to digital and the design opportunities and implications that have accompanied this move. There is much of significance to discuss within just this one topic but there have been a number other key developments and these are described and discussed in near chronological order.

\section{The Theremin}

Invented by Leon Theremin in 1920, the theremin (originally called the thereminvox) was designed to be played using two hands moving within weak electromagnetic fields generated around two aerials; one controlling pitch and the other volume. Although it is often cited as being the earliest fully electronic instrument, in fact most noticeably an instrument called the Telharmonium was patented in 1896 (Holmes 2002) and therefore predates the theremin by over twenty years. However, this instrument, other than being as large as a church organ when fully realised in 1906, produced its sound via electromechanical means using 'tone wheels' and is often cited perhaps more accurately as being the precursor to the Hammond organ. The theremin was very limited in tonal variation but provided considerable richness in terms of expressive freedom. It emitted a constant tone, with the frequency of the pitch produced being related to the distance between the performers hand and the aerial controlling pitch. Moving the hand slowly from one location to another would produce a glissando effect; rapid small movements back-and-forth would produce vibrato. Similar movements with the other hand would produce either a gradual change in volume or a tremolo effect.

The notion of 'inclusive design' is a modern concept and it is quite unlikely that the design process behind the original theremin had much concern for whether the instrument would be easy to use for musicians with disabilities. However, the theremin is particularly accessible. Although the original instrument was designed to be played using the hands, realistically any body part can be used; it is just as feasible to control a Theremin with a foot (leg, arm) as it is with a hand. In terms of dexterity, the instrument is quite forgiving, small errors in judgement are easily absorbed into the quality of the instrument's natural sound. Also, the mapping of movement to sound is very intuitive making the theremin relatively easy to understand. More significantly, its mode of operation has inspired a number of theremin-like enablingtechnologies that are more keenly focused on the potential use of such devices by disabled musicians. Discussed later in this article, one or two of these 'touch-free' technologies have become almost standard accessories for use within special needs in addition to more occasional use of the theremin itself (Magee 2006). 
There may appear to be little of immediate interest when considering the relative merits of keyboard instruments over other musical instruments in terms of general levels of access. After all, most instruments require considerable control of fine motor skills such that where one piece of technology is difficult for an individual to work with, many others are likely to share similar requirements in dexterity. All instruments have their individual merits though (e.g. range, tone, ease of use, flexibility, portability etc.) and the traditional keyboards tends to excel in both polyphony and range. It is also fair to say that where some instruments require considerable cognitive and/or physical effort to produce a note, the keyboard has a large range of pitches available, directly in front of the musician and ready to use. The gradual move from mechanical to electronic sound production in keyboards provided yet another opportunity, that of emulation. Even with early electronic organs, a mix of appropriate drawbars could synthesise various woodwind and bowed string instruments. So, the general accessibility afforded by the layout of the keyboard is enhanced further if it is considered as a potential gateway to the electronic emulation of any number of other instruments. If mastering a particular instrument is beyond the physical abilities of a particular individual then perhaps the keyboard can provide access to similar sound but by means of a (potentially) more usable interface.

\section{MIDI}

First published in October 1983, the main aim behind the introduction of the Musical Instrument Digital Interface (MIDI) standard specification was to allow electronic instruments from different manufacturers to communicate with one another. Although MIDI originally blossomed within the keyboard-based market allowing the keyboard to operate independently of a specific sound module, this success continued into most aspects of music production and performance. Perhaps most noticeable was the potential to record and edit MIDI messages by way of a sequencer. Using only limited computing power and memory, complex compositions could be achieved as the actual sounds were stored or produced via external devices. Originally hardware in implementation, such MIDI 'sequencers' rapidly moved into the software domain and it is here that MIDI presented its real potential. Software programmers could design software that allowed the user to work at differing levels of complexity including the opportunity to create music at a phrase-based level. Instead of a single action (e.g. pressing a note, hitting a pad) resulting in a single sound, the same action could trigger any number of algorithms that would produce complex tonal, harmonic and/or rhythmic patterns. Effectively, music could be composed and performed at differing degrees of abstraction ranging from triggering specific events in real-time to, triggering individual phrases in sequence or in real-time, to triggering pseudo-random variations or mutations of phrases and even to working at an entirely conceptual and perhaps rule-based compositional level. In short, very simple input could be used to create very complex music in a very controlled way and, potentially, in real-time. Indeed, this has led to some quite inspirational and novel approaches to music making aimed at musicians and music-learners with disabilities. Anderson (1999) describes various approaches to novel interaction in this area but some of the more complete and commonly used systems are discussed later in this article. 
The arrival of mainstream digital hardware sampling technology in the early 1980s significantly enhanced the potential for adopting a one-instrument-accesses-all approach to music performance. There are obvious creative advantages to be gained from employing sampling technology as a single musician can access a virtually limitless array of realistic instrument sounds. This is illustrated quite clearly in the performances of one particular teenager who has been working with the Drake Music Project. She uses a combination of small but precise head movements (captured by a motion sensor) along with two switches for her thumbs to produce MIDI notes that are mapped to different sampled instrument sounds. This combination of technologies enables her to perform pieces for instruments that she would not normally be able to access (an example of one her performances, a piece for cello, is currently available via the internet (see Charlotte)).

At another level, sampling technology also creates possibilities for working with any 'real' sound captured in digital format. This presents further opportunity for composing and performing music at a higher phrase-based level as whole sections of sampled and possibly quite complete music can be triggered, layered and looped. Coupled with MIDI to trigger any combination of sampled sections, loops and MIDI phrases it is not difficult to comprehend why the commercial market has expanded so rapidly in this area. In effect, anyone can create music without necessarily being able to play a specific instrument. This is just as true for the ordinary user as well as those with extraordinary needs but with a significant difference of size of consumer group.

Analogue and digital recording equipment

Some of the developments and concepts outlined so far are echoed in the nature of recording and production equipment. Computer-based approaches to recording in the digital domain have led to an understandable move from large, analogue, dedicated consoles for recording and processing the sound of physical instruments to smaller, digital, self-contained, multifunctional systems that facilitate any number of virtual instruments and off-board processors. In terms of accessibility, the move from big to small can have distinct advantages, particularly for individuals with limited mobility, as can the general move into the computer-based domain where adaptive technologies can often be used to enhance access according to individual needs. There are costs though. An obvious way to reduce overall size is to reduce the size of individual components: large faders, switches and knobs become small faders, switches and knobs, and subsequently, can become that bit more difficult to manipulate where dexterity is an issue. Likewise, where an appreciation of spatial-layout is important, such changes can quickly lead to a cluttered control surface. Another method for saving space is to increase the functionality of individual controllers. Why have many faders, switches and knobs when a smaller number of assignable controls will do? This is an area that Norman (1998) discusses within a context of user-centred design identifying that designs that rely on less controls can lead to the false perception of a system being easy to use because it looks simple. He suggests that a system is actually more likely to be easy to use if the number of controls matches the number of functions available. If multiple modes are employed, the mode in which a particular controller is operating will generally be shown on a visual display, a common design 
feature yet one that might make a particular piece of technology completely inaccessible for a visually impaired (VI) user.

\section{Current Technologies, Accessibility and Education}

So, how might some of these commonly used technologies fair when scrutinised within a British context of meeting the requirements of the Special Educational Needs and Disability Act of 2001? In effect, this act amended the Disability and Discrimination Act of 1995 to include more specific requirements for education providers. There are two key inclusions aimed at further and higher education providers with similar, but separate, inclusions aimed at schools. In brief, these state that there is:

a duty not to treat disabled students less favourably, without justification, for a reason which relates to their disability; and a duty to make reasonable adjustments to ensure that people who are disabled are not put at a substantial disadvantage compared to people who are not disabled in accessing further, higher and LEA secured education. (HM Gov, 2001)

The key phrase in this statement is 'reasonable adjustment' as this quite simply means that if there are alternatives modes that can be used for communicating ideas or demonstrating techniques, modes that are perhaps more appropriate to an individual's specific needs, then these should be made available. This creates one or two dilemmas for education providers. Perhaps most apparent is the desire for FE and HE institutes to be seen as operating in-line with industry standards. For example, ProTools is widely accepted as being an industry standard studio recording technology and it is not unlikely that technology of this type will form the basis for studio recording practical sessions. From a creative perspective, ProTools is a powerful softwarefronted recording environment which, as Savage and Challis (2001) have observed, is also intuitive enough for children as young as nine years old to work with creatively and independently. Unfortunately, and in stark contrast, it is also a particularly inaccessible environment for VI users to work with (Zahradnicky et al., 2008).

A second dilemma faced by some educational institutes is how to provide a practical experience for a large volume of students? As Boehm (2007) observes, the general increase of HE music-related courses that incorporate both creative and technological elements can lead to courses being shared between different faculties; faculties that may operate on highly contrasting models of class-size. One resource that is likely to be in high demand is the recording studio. The very nature of the task, and size of environment, dictates the involvement of perhaps only a few people in a process that can sometimes be very long. There is obvious potential for this aspect to impact upon the experience of students studying, for example, sound-engineering. However, as Eno (2004) reminds us, the recording studio is not the exclusive domain of the sound-engineer, and should clearly be seen as a compositional tool. So, this same level of high demand is also likely to impact upon the experience of those studying popular music composition.

An alternative (or possibly additional) experience can be offered using computers running popular software as recognised earlier (Cubase, Sibelius etc.) with perhaps a small collection of 'plug-in' virtual instruments. In practical terms this 
might be seen as a flexible, and therefore sensible way, of providing access to a wide variety of sound sources. The potential follow-on effect in terms of accessibility though, is that the requirement for physical instruments can be reduced, resulting in more computer-based emulation. There are already a number of recognised design issues here for users of software instruments and recording environments (Magnusson 2006), not least that the two-dimensional screen is simply not an efficient way of navigating and controlling the number (and type) of physical controls that often need to be available. This is before any consideration has been given as to whether the user might have additional needs. For VI students in particular, highly complex graphic environments simply do not lend themselves to access via screen-reading software such as JAWS or HAL. Where standard text is not available for the screen-reader to access, graphic objects need to be interpreted within an off-screen model such as that described by Kochanek (1994) where recognised items can then be referenced using a scripting system. Obviously, this kind of adaptive software and hardware intervention can only really be achieved if the manufacturer of the original product is willing to collaborate at some level.

At this point, it is also perhaps worth considering the role of common music notation within Popular Music. Moore (1993), reminds us that the "primary medium of transmission of rock, since at least mid-1950s rock ' $n$ ' roll, has been the recording" suggesting that where a notated rock-score exists it will probably be transcribed. He does acknowledge, however, that notation has a valid place within popular music and cites the continued use of sheet music as evidence of this. Indeed, in the UK, the Rockschool graded exam series includes notated pieces and technical exercises. These qualifications are included within the UCAS tariff for evidencing musical ability (see UCAS). Graphical notation of this type is clearly difficult for VI students or pupils to access and it is likely that the same is true for dyslexics. Although there are non-visual alternatives, such as Braille music and 'Talking Scores', these have inherent complexities of their own; Ockelford (1996), presents a detailed overview of both Braille music and 'Talking Scores'. In brief, both are 'displayed' in a serial fashion with the reader being required to 'read' everything before effectively filtering out any unwanted information. Within this, there are fundamental conflicts between Braille and Braille music; the Braille codes for the musical letters A to $G$ do not correspond to standard Braille for the same letters. In fact, they map onto the Braille letters for I J D E F G H; a potential source of confusion for those learning both systems simultaneously. Also, not as many registered blind people read Braille as might be imagined, this figure may be as low as only 2\% (Bruce, McKennell and Walker 1991).

This discussion is on the role of technology and accessibility though, so what technological aids to accessing music notation are available to VI music learners? Within academic research there have been a number of projects that have addressed this area. Challis and Edwards (1997) proposed and prototyped a system that would allow VI music learners to browse a tactile structural-overview of one or more pages of music. Using the 'Weasel' system (Challis 2000), music learners could press onto touch-responsive tactile-overlays containing aspects of musical structure (bars, repeats, dynamics etc.) and directly select extracts or phrases for immediate display. Filters could also be applied to enable the reader to focus in on key music learning tasks (pitch, rhythm, fingering etc.) with the notation being displayed as audio playback, speech or potentially a refreshable Braille display. Other non-visual 
approaches have since been considered by McKenzie and Marwick-Johnstone (2008) and also by Nicotra and Quatraro (2008). However, accessibility to commercially computer-based notation platforms (e.g. Sibelius, Finale) is currently very limited (McCann 2000). Although screen reading scripts for older versions of Sibelius (v.3) have recently been made available, the more current versions (v.4 and v.5) are, as yet, unsupported.

Issues over interaction in complex graphical software are not limited to VI users, the on-screen interface can tend to be difficult to manipulate for sighted users who have limited dexterity. Access by keyboard short cuts may be available for many actions but the tendency to rely on floating toolboxes will usually require the use of a mouse, the control of which can be an issue by itself. Switch-based access is almost impossible. The issue of how accessible technology should be from a commercial perspective will carry on for many years to come but that is not the central issue here. The real question is whether reasonable adjustment can and should be made? There are without doubt alternative modes for teaching and exploring the general concepts being covered within most popular music and music technology courses in further and higher education. So, the real issue is whether the discipline being taught can be achieved through other means and the answer is almost certainly yes.

\section{Novel Instruments and Interfaces}

The following sections detail a variety of commercial and research-based interfaces that in one way or another provide enhanced accessibility within a music performance setting. Much of the focus here will be on two particular areas: the use of Sounbeam by the band 'Quinta Punkada' and personal research into an accessible musical instrument. These are followed by short discussions on a number of other technologies that are in some way enabling. This is not an exhaustive list and there are many live research projects exploring practical approaches to novel interfaces in music making. (The New Instruments for Musical Expression (NIME) conference held annually since 2001 is just one forum where researchers, designers and performers share their ideas for new approaches to digital musical instrument design. ArtAbilitation is another similar conference-based forum, but one that deals more exclusively with disability, technology and the arts.)

During these discussions, it will at times be useful to consider such novel technologies in terms of the different modes of performance behaviour they can facilitate. A framework for this is described by Malloch et al. (2006) who suggest three such modes of performance behaviours: skill-based, rule-based and modelbased. Of these, the mode of musical interaction-behaviour most similar to that of playing a conventional musical instrument lies within the skill-based domain. The implication is that the user will be interacting in real-time in response to a continuous audio-stream. The other two models of musical interaction-behaviour operate at increasingly abstract levels of interaction with the user's interaction being less and less involved in terms of overall control and 'connection' with the sounds being made.

\section{Soundbeam and Quinta Punkada}

Soundbeam was originally designed to be used by dancers but has since become increasingly popular as a method of making music performance accessible to 
individuals with specific needs. Swingler (1998) describes the system as being "an invisible, elastic keyboard in space that allows sound to be created without the need for physical contact with any equipment". Soundbeam is popular in schools with special needs provision and is also generally acknowledged as being useful within both community music (Healey 2005) and music therapy (Magee 2006). The system can accommodate different types of switches and sensors, but the primary source of input comes from an ultrasonic 'beam' as the name suggests. When this beam is interrupted, sounds are triggered in much the way a theremin operates. Though, in contrast to the gliding-pitch sound of the theremin, Soundbeam maps musical scales in steps along the beam using MIDI notes. These are transmitted to any sound-module that is attached, so the diversity of sounds available for the performer is potentially very rich. The length of the beam can be varied according to an individual's needs or to accommodate environmental constraints. Interaction with the beam is essentially one-dimensional as movement within the beam cannot be sensed at multiple locations. However, the system adopts rule-based behaviours to allow the triggering of predefined harmonies and musical patterns so that polyphonic output can be achieved i.e. chords.

Soundbeam is frequently featured in workshops and events hosted by Drake Music Project (UK) but there has been some truly inspirational performance work achieved by the Portuguese pop-rock band 'Quinta Punkada'. Based at the Cerebral Palsy Association College in Coimbra (Portugal), and coordinated by Paulo Jacob. Quinta Punkada are a group of musicians who play and perform publicly despite mobility issues that would normally make access to many conventional instruments difficult if not impossible. Embracing many styles (including hints of jazz, blues and even punk) the band consists of keyboards, drums, vocals and one or more Soundbeams. Perhaps the most noticeable component within the sound of Quinta Punkada is afforded by Soundbeam. Using one as a lead instrument, the band's 'soundbeamist' will occasionally break into a 'guitar' or 'keyboard' solo, gesturing with his hand or arm within the beam. Mapped to a blues scale, a sweeping gesture allows him to glissando up or down the pitches with smaller movement producing trills and arpeggiated effects. Three out of the four regular members of Quinta Punkada are wheelchair users, each with quite profound mobility problems. Indeed, the very notion of attempting to play a conventional guitar or keyboard would simply be unrealistic. Using this enabling technology, the same individuals are presented with the means to improvise and express themselves freely in a way that is both natural and fitting within a popular music context.

Consider the notion of improvising with a conventional instrument above a 'groove'-based chord backing. Although, a rich and expressive solo might be achieved through the exploration of only a limited pitch-set (perhaps a single pentatonic scale), the player must still demonstrate skill in avoiding the pitches that do not belong within the set. With this in mind, devices like Soundbeam can introduce a level of 'compensation' into the interaction process. Though, as Healey (2005) reminds us, the concept of ownership is important and, in this respect, there can be the potential for the relationship between performer and sound to be compromised. However, with careful composition and arrangement, a player can perhaps simply be liberated from the worries of skill-based errors that could result in mis-hit notes, encouraging the exploration of runs and phrases that would ordinarily be very difficult to achieve. In this instance the technology is presenting new opportunities and the 
performer is not relinquishing control within the decision making process. The solos of the lead-instrumentalist in Quint Punkada pay testament to this. These are not simplified, limited solo passages, they are exciting, expressive and complete.

\section{The Benemin}

There are a number of novel musical instruments and controllers that have emerged from within academic research and some of these are discussed later in this article. One such device is the "Benemin" an accessible musical instrument that has been designed and built as part of ongoing personal research into the area of accessibility and music. The rationale for the prototype Benemin was realised through feedback from educators which suggested that there could be a place for a dedicated 'instrument' to be available within special needs education; this being in addition to very flexible systems such as Soundbeam. It was recognised that not all educators involved with music activities in special needs education are trained musicians and as such did not always feel comfortable or confident with setting up and operating systems that may, at first, appear complex. With this in mind, the central aim of this project has been to design an accessible controller that is a simple diatonic instrument when operated in its default (or start-up) mode, even though additional advanced features may be available. The idea of mapping gestures onto sound was seen as both powerful and potentially intuitive so interaction of this type has been preferred but with an added desire to respond to more than one dimension of movement. Additional feedback suggested that if overall cost could be kept low, this could increase the potential for a number of instruments to be available. This was understood to be advantageous as the instrument would not then be regarded as a 'special' resource that only had limited access.

As a first solution to these suggestions, the prototype Benemin is an array of eight distance-measuring infra-red sensors that allow a performer to trigger sounds via MIDI. There is an obvious similarity between this instrument and Soundbeam as both employ distance measuring technology to map movement onto sound and music. However, the Benemin is effectively polyphonic. Gestures can be made with two hands or arms above the instrument (up to a range of about $80 \mathrm{~cm}$ ) to produce simple harmonies. Potentially, all eight sensors can be triggered simultaneously. More detailed accounts of the design and construction of the Benemin can be found elsewhere (Challis and Challis 2008, Challis and Smith 2008) but it is perhaps important to appreciate that it operates at two levels of complexity: as an instrument (to meet the requirements just outlined) and as a controller (to allow more complex control of external software).

The instrument is not aimed exclusively at users with special needs, instead it is seen as being an instrument for use by anyone but where the design process has included accessibility as a key consideration. So, the instrument is being tested within a variety of settings including, free improvisation, community music, special needs education and general music education. The instrument can already be seen in use as part of free-improvisation (Challis, Smith and Wiblin 2008) but for the purposes of this article it will be of most interest to reflect on the initial findings from its use within special needs education._Currently, the Benemin is being assessed by three contrasting user groups in special needs schools in the North of England. Within these groups, it is being considered firstly as an 'inspirational' device where individuals 
with severe learning difficulties can benefit from musical reinforcement within causeand-effect style learning patterns. The second user group includes individuals with mild learning difficulties and some mobility issues and the final group includes individuals whose main barriers are related to mobility.

So far, the majority of testing has been with individuals from the second user group and the initial results are promising. Pupils have been allowed to explore the instrument in their own time, in effect, allowing them to freely improvise, exploring textures and patterns of their own creation along the way. There have been two particularly rewarding observations made during this early testing. Firstly, players will tend to instinctively use more than one hand to produce harmonies in an intuitive way. Secondly, having the instrument constantly available and ready for anyone to try, appears to encourage individuals who may be quite reserved to engage with this process of exploration in their own time and on their own terms. Although, none of the children were working within a group setting as such, there have been examples where they have spontaneously responded to other musical activities occurring elsewhere in the classroom. It was also clear that the diatonic nature of the instrument would enable their improvisations to be incorporated into structured backing of some description; this still needs to be explored in practice though. The Benemin project is still in its infancy and further testing and subsequent redesigns are being planned and coordinated for the forthcoming year.

\section{Sensor-based Technologies}

Novel approaches to sound production tend to involve novel technologies. Infrared and ultrasonic distance detectors, tilt and vibration sensors, accelerometers, pressure and heat sensors and even flex-sensors (to detect bending) are all easily available from online sources and at a cost that has allowed DIY music technologists and circuit-bending enthusiasts to construct all manner of unusual music making devices. Comprehensive overviews of the types of sensors and control technologies available and how they might be incorporated within novel performance devices are presented by O'Sullivan and Igoe (2004) and also by Miranda and Wanderley (2006). It also seems that the commercial market has responded to this interest to a degree partly by following suit and incorporating similar technologies into mainstream design and partly by offering opportunities for enthusiasts to design and build their own systems. One such company is Doepfer Musikelektronik which manufactures a selection of modules specifically aimed at DIY music technologists._Whether this has influenced the mainstream market to reconsider exactly who uses what (and how) musically is difficult to judge but there has been a distinct increase in design considerations that appear to be aimed at making music more accessible to all. It could go without saying that this is probably a good thing and some of this technology has direct application for beginner and professional musicians alike. However, the caveats outlined earlier in regards to market forces remain true, so it is also worth remembering that 'novel' does not mean always mean 'accessible' and that the likely target users for most commercial products are almost certainly those without any significant disabilities.

\section{Theremins and the Etherwave}

Described at the beginning of this article, the theremin has experienced enough of a revival in popular music circles to be deemed worthy of renewed commercial 
production on a large scale. The Moog Etherwave is probably the most notable in this respect, being an adaptation of the original design and adding only a few enhancements to the functionality of the original (skill-based) instrument. There appears to be considerable credibility afforded through the use of a theremin in popular music and it may well be that this has evolved partly through the obvious association of the instrument with cult science-fiction soundtracks. Putting aside the exact origins of this acceptance, here is an instrument that has featured in the music of Led Zeppelin, Goldfrapp, Marilyn Manson, Terrorvision, David Gray, Gomez and Timbaland to name but a few.

\section{KaossPad}

The KaossPad from Korg is now in its third version. Originally aimed at the dance and DJ market, the pad is essentially a sophisticated sound processor and phrase sampler. The user can manipulate sound in all manners of creative ways using an interface that employs a few buttons and a touch sensitive area where gestures can be made using a fingertip. Perhaps the most significant feature of the KaossPad in terms of accessibility is that there are few hidden features, most of the functionality of the controller is available from the touch-sensitive area and the surrounding buttons which mainly have dedicated functions. It is a noticeable strength of the device that it can create rich and complex sounds based on really quite small movements.

\section{Handsonic}

Roland's Handsonic is essentially an electronic hand-percussion synth-module. Technology of this type that facilitates the triggering of sounds via 'pads' is occasionally used within music therapy (Magee 2006) and has obvious potential within community music. This unit is small and light enough to be placed on a performer's lap but can also be mounted on a stand, making it particularly convenient to set-up for wheelchair users. Up to fifteen pressure sensitive pads can be accessed within a relatively small surface area giving access to a variety of hand-percussion sounds. There are also tuned-percussion sounds available with a chromatic scale of one octave mapped across the various pads. In addition to the pressure sensitive pads, there are also two ribbon-sliders and a D-beam which makes use of an infra-red distance measuring device; all of these can be used to trigger or modify sound.

\section{MIDI Creator}

Originally developed at the University of York, MIDI Creator (Abbotson et al. 1994) is now a commercially available MIDI controller that allows a variety of sensors to be used to capture a user's actions which are then mapped onto MIDI messages (see MIDI). The device is aimed almost entirely at individuals with special needs and has been much used by the Drake Music Project. The types of sensors available include, pressure mats, ultrasonic-beams, squeezable pressure-sensors, capacitive devices and accelerometers. Sensors are either discrete or proportional and are mapped onto userdefinable scales and chords. However, the technology also functions most effectively as an interface to various software environments (e.g. E-Scape). 


\section{Tenori-On}

Developed in partnership between Yamaha and media artist Toshio Iwai, the TenoriOn is perhaps one of the most novel approaches to music making and interaction currently available on the commercial market. A sixteen by sixteen grid of ledswitches allow a user to set and interact in real-time with musical events that are driven by an internal sound generator. Part rule-based and part skill-based, the TenoriOn has obvious potential for people who find conventional musical instruments and associated equipment physically demanding to work with. The instrument is selfcontained, compact, lightweight and easy to access (in terms of functionality) yet offers considerable richness in terms of both musical textures and performance interaction. It is already gaining credibility in popular music performance and has featured recently in performances by Bjork.

\section{E-scape}

In contrast to conventional approaches to composition through sequencing, E-scape (Anderson and Smith 1996) introduces another dimension as a user can also elect to be a conductor/performer. Although it could be argued that there are similar opportunities within all sequencer-style programs (e.g. dynamically introducing and removing explicit tracks in real-time), within this specific environment there is a significant level of additional emphasis attached to how and when events are played. Using a track-based, events-against-timeline approach that will already be familiar to most composers and musicians, the E-scape environment allows a user to input or record events that can then be played back. However, it is possible to scroll through events on a track by moving the mouse or pressing switches, in this respect the software offers a skill-based musical instrument facility within a rule-based musical environment. Designed by the Drake Music Project with users with specific needs in mind the system can be controlled (and therefore, 'played') using novel interaction devices of the types discussed earlier e.g. Soundbeam and MIDI Creator.

\section{MIDIGrid}

Originally developed at York University, MIDIGrid (Hunt, 1988) adopts a phrasebased approach to music composition and performance aimed at widening access to music through the use of easily available technology. The concept is simple, a computer screen is divided up into a grid of cells that are variable in size. Each cell can contain anything from a single note up to a complex melodic, harmonic or rhythmic idea. Cells are then triggered by moving a mouse, a trackball or a joystick. As with E-scape, the user is offered a skill-based instrument that is constrained within a rule-based environment. However, this particular system can afford richer levels of interaction within an improvisational context than those offered by E-scape having up to two hundred cells available within a single grid. Where E-scape functions mainly as a compositional tool whilst also facilitating performance, MIDIGrid is probably best thought of as being predominantly a performance tool.

\section{Summary}

In terms of accessibility, it would appear that the range of typical music production provision available in most educational institutes might be compromised by an 
underlying need to be visibly in-line with perceived technological standards as dictated by current industry practice. Although there is a legal requirement in Britain for providers of education to make reasonable adjustment according to individual needs the means by which this is achieved may well not be obvious within, for example, a course prospectus. With this in mind, it is possible that many popular music, music production and music technology courses may not appear to be particularly inviting from the perspective of applicants with additional needs.

Until recently, there was a freely available software application (Bobby) ${ }^{8}$ that would assess the accessibility of a website. If deemed to be accessible, the site could then display the Bobby logo. A site with the Bobby symbol was effectively saying 'welcome' to most users with specific needs. However, there was another message implicit within the use of the Bobby logo as the likelihood of meeting the requirements for Bobby's assessment was that the site would probably not appear to be as 'slick' as that offered by others. Therefore, the Bobby logo was also representing a site-owner's order of values; accessibility to the site's information was valued higher than the site's immediate appeal. As yet, there seems to be no parallel that exists within education, and in particular, for provision that is measured by a perceived need to be visibly industry-aware. There is equipment and software available that is accessible and pedagogically appropriate, so, why rely on a particular piece of recording software and a digital desk when a different piece of software and an analogue desk will be accessible to all? Why use a specific software sequencer that is particularly inaccessible in place of one that is accessible? Why restrict the music making experience to mainstream instruments when there are so many novel alternatives?

The failure to inspire individuals with specific needs to explore alternate possibilities for music-creation in this way could be a contributing factor to the noticeable under-representation of musicians with disabilities within our current popular-music industry. Providers of education should consider evaluating the perceived message that is currently being sent and look to establish a way of stating that 'access-for-all' is still a key value within music-education.

\section{References}

Abbotson, M., Abbotson, R., Kirk, P. R., Hunt A. D. and Cleaton A. 1994. Computer music in the service of music therapy: The MIDI Grid and MIDI Creator systems. Medical Engineering Physics, Vol. 16, May 1994, pp253.

Anderson, T. 1999. 'Using music performance software with flexible control interfaces for live performance by severely disabled musicians' in Proceedings of $25^{\text {th }}$ Euromicro Conference.

Anderson, T. and Smith, C. 1996. 'Composability: widening participation in music making for people with disabilities via music software and controller solutions' in proceedings of ASSETS '96.

Boehm, C. 2007. 'The discipline that never was. Current developments in music technology in higher education Britain' in Journal of Music, Technology and Education, Vol. 1, Num. 1, Intellect Ltd. 
Bruce, I., McKennell, A. and Walker, E. 1991 'Blind and Partially Sighted Adults in Britain: the RNIB Survey’ Her Majesty’s Stationary Office, London.

Challis, B. P. 2008. 'Applications for Proximity Sensors in Music and Sound Performance' in Proceedings of 11th International Conference on Computers Helping People with Special Needs, Springer-Verlag (Heidelberg).

Challis, B. P. and Edwards, A. D. N. 1997. 'Non-Visual Music Representation: A Computer Based Approach’ in Interfaces (35) Summer 1997. Bulletin of The British HCI Group

Challis, B. P. and Edwards, A. D. N. 2000. 'Weasel: A System for the Non-Visual Presentation of Music Notation' in Proceedings of ICCHP 2000.

Challis, B., Smith, R. and Wiblin, I. 2008. Untitled. Open Ear, Cardiff, http://openear.wordpress.com/

Charlotte http://uk.youtube.com/watch?v=sNBhIZCL54Q

Doepfer Musikelektronik. http://www.doepfer.de/home_e.htm

Drake Music Project. http://www.drakemusicproject.org.

Eno, B. 2004 'The Studio As Compositional Tool' (reprinted article from Downbeat July/August 1983) in Audio Culture: Readings in Modern Music, Eds. Cox, C. and Warner, D. Continuum.

HM Gov. 2001. 'Explanatory Notes to the Special Educational Needs And Disability Act 2001', chapter 2 of part 2, ISBN 0105610011.

Holmes, T. 2002. 'Electronic and Experimental Music', pp44-65, Routledge.

Hunt, A. and Kirk, P.R. 1988. 'MIDIGRID - A New Musical Performance and Composition System' in Proceedings of the Institute of Acoustics.

Kochanek, D. 1994. 'Designing an Offscreen Model for a GUI' in International Conference on Computers for Handicapped People '. Springer Verlag (Berlin).

Magee, W. 2006 'Electronic technologies in clinical music therapy: A survey of practice and attitudes' in Technology and Disability, Vol. 18, pp139-146. IOS Press.

Magnusson, T. 2006. 'Affordances and constraints in screen-based musical instruments' in Proceedings of the 4th Nordic conference on Human-computer interaction: changing roles. Oslo, Norway. ACM.

Malloch, J., Birnbaum, D., Sinyor, E and Wanderley, M. 2006. 'Towards a New Conceptual Framework for Digital Musical Instruments' in Proceedings of the $9^{\text {th }}$ International Conference on Digital Audio Effects. 
McCann, W. 2000. 'The Dancing Dots music editor: a non-graphical approach to scoring for the blind musician or dyslexic' in Proceedings of CSUN Conference on Technology and Persons with Disabilities (2000).

McKenzie, N and Marwick-Johnstone, B. 2008. 'Making the I-Maestro Music Learning Framework Accessible' in Proceedings of 11th International Conference on Computers Helping People with Special Needs. SpringerVerlag (Heidelberg).

MIDI Creator. http://www.experia-innovations.co.uk

Miranda, E and Wanderley, M. 2006. 'New Digital Musical Instruments: Control And Interaction Beyond the Keyboard'. A-R Editions.

Moore, A. F. 1993. Rock: The Primary Text. Open University Press.

Nicotra, G. and Quatraro, A. 2008. 'CONTRAPUNCTUS Project: A New Computer Solution for Braille Music Fruition' in Proceedings of 11th International Conference on Computers Helping People with Special Needs. SpringerVerlag (Heidelberg).

Norman, D. A. 1998. 'The Design of Everyday Things', pp208-209. The MIT Press.

O'Sullivan, D and Igoe, T. 2004. 'Physical Computing'. Thompson Course Technology.

Ockelford, A. 1996. 'Music Matters' Royal National Institute for the Blind (RNIB) ISBN1-85878-071-3

Pitts, A. and Kwami, R. M. 2002. 'Raising students' performance in music composition through the use of information and communications technology (ICT): a survey of secondary schools in England' in British Journal of Music Education, 19, 1, pp61-71. Cambridge University Press.

Savage, J and Challis, M. 2001. 'Dunwich revisited: collaborative composition and performance with new technologies' in British Journal of Music Education, Vol. 18, Num. 2, pp139-149. Cambridge University Press.

Soundbeam. http://www.soundbeam.co.uk

Swingler, T. 1998. “"That Was Me!": Applications of the Soundbeam MIDI controller as a key to creative communication, learning, independence and joy' in Proceedings of CSUN Conference on Technology and Persons with Disabilities (1998).

UCAS. http://www.ucas.ac.uk/students/ucas_tariff/tarifftables/\#music 
Zahradnicky, T. Lorencz, R. and Musil, P. 2008. 'Making ProTools Accessible for Visually Impaired' in Proceedings of 11th International Conference on

Computers Helping People with Special Needs. Springer-Verlag (Heidelberg). 